\title{
Alimento Funcional: Uma Nova Abordagem Terapêutica das Dislipidemias como Prevenção da Doença Aterosclerótica
}

\author{
Professora Adriana D‘Auria Aparecida Braga (Nutrição - UniFOA) - \\ adri.nutri@uol.com.br \\ Acadêmica Valéria Calmeto Noronha Barleta (Nutrição - UniFOA) - \\ valeriabarleta@globo.com
}

\begin{abstract}
Resumo
A nutrição é uma das ciências mais antigas que visa estudar o alimento e as modificações que este sofre ao passar pelo organismo. No Japão na década de 80, estudos comprovaram que certos alimentos além de suas funções básicas nutricionais demonstraram benefícios fisiológicos e reduziram o risco de doenças; foram denominados alimentos funcionais, que podem potencialmente reduzir a ocorrência de doenças cardiovasculares, cânceres, osteoporose, problemas intestinais. A associação Americana do Coração já indica o uso de alimentos funcionais como parte da dieta para dislipidemias, o principal fator desencadeador da aterosclerose. As doenças cardiovasculares lideram o índice de mortalidade no Brasil, e são responsáveis por 50\% das mortes nos EUA. Os principais compostos ativos responsáveis pelas ações preventivas das doenças cardiovasculares pertencem ao grupo dos polifenóis (flavonóides e isoflavonas), carotenóides e ômega-3; encontrados em alimentos usuais como: alho, tomate, berinjela, chocolate amargo, uva - vinho tinto, peixe, oleaginosas, chá verde, soja, especiarias, leguminosas, cogumelos e maçã. Hoje os alimentos com atividade funcional fazem parte da nutrição aplicada no dia-a-dia de muitos nutricionistas, nas mais diversas atividades,exigindo do profissional um aprofundamento na ciência dos compostos funcionais.
\end{abstract}

Palavras-chave: alimentos funcionais; doenças cardiovasculares; dislipidemia.

\begin{abstract}
Nutrition is one of the most ancient science that aim studying food and its modification during the ingestion process. In Japan during the 80's, searches confirmed that some foods besides basic nutritional function showed physiological benefits and reduce the risk of diseases. They were called Functional Foods, which may reduce risk of Coronary Heart Disease (CHD ), cancer, osteoporosis, intestinal problems. The Heart American Association has
\end{abstract}


already used functional food, as part of diet for dyslipidemia, the main factor of development arteriosclerosis. The Coronary Heart Disease (CHD) is the main cause of death in Brazil, and it is responsible for $50 \%$ of deaths in the U.S. The main active component responsible by CHD preventive actions are the groups of polyphenol (flavonols and isoflavonols), carotenoids and Omega 3, provided from some usual food such as, garlic, tomatoes, eggplant, chocolate, grapes, fish, walnuts, green tea, soy, spice, leguminous, mushroom and apple. Nowadays food with functional active make part into many nutritionist routine in different ways, that's the reason for many professional take more knowledge related to functional components.

Key words: 1) functional food; 2) Coronary Heart Disease; 3) dyslipidemia.

\section{Introdução}

O presente trabalho versa sobre o uso de alimentos funcionais associados à terapia nutricional convencional das dislipidemias em pacientes com risco aterosclerótico; mostrando que sua utilização pode prevenir e tratar as dislipidemias, reduzindo os casos de doenças ateroscleróticas, que acometem grande parte da população mundial, engrandecendo ainda mais o papel da nutrição na atuação dietoterápica. (ACCIOLY,2002)

A nutrição é uma das ciências mais antigas que visa estudar o alimento e as modificações que este sofre ao passar pelo organismo, sua antiguidade é comprovada a partir das citações de vários autores: Hipócrates há 2500 anos advertiu sobre a importância da relação entre nutrição e doença; Lavoisier no ano de 1770 desvendou a combustão de alimentos e respiração celular; Pasteur entre os anos de 1857 e 1890 afirmou sobre a necessidade de aprofundar o estudo dos alimentos; Benedict no ano de 1919 constatou mudanças fisiológicas em relação à alimentação; e Pedro Escudeiro, considerado o pai da nutrição, no ano de 1937 introduziu o estudo da alimentação e nutrição nas escolas de medicina de seu país de origem, Argentina. (TIRAPEGUI,2002)

Nas últimas décadas vários estudos têm demonstrado a associação entre dieta e doenças crônico-degenerativas, atribuindo aos alimentos funcionais a capacidade de proporcionar benefícios à saúde, além daqueles conferidos pelos nutrientes presentes nos alimentos, proporcionando a fusão da dieta terapêutica convencional com uma conduta dietoterápica funcional, levando em consideração a capacidade dos alimentos funcionais de reduzir o risco de ocorrência de doenças crônico degenerativas.(PASCHOAL,2001) A associação Americana do Coração já indica o uso de alimentos funcionais como parte da dieta para dislipidemia.(BRICARELLO,2001)

As doenças cardiovasculares representam a principal causa de morbidade e mortalidade no mundo, (ACCIOLY,2002) sendo a aterosclerose considerada um problema de saúde pública, (FERNANDES,2004) por ser uma doença que leva ao espessamento da parede e diminuição da elasticidade vascular, a aterosclerose é a principal causa que 
predispõe infartos do miocárdio, acidentes vasculares cerebrais, doenças vasculares periféricas. As doenças cardiovasculares lideram o índice de mortalidade no Brasil segundo dados do DATASUS (pesquisa do Sistema Único de Saúde Brasileiro), (PRADO,2001) e também são responsáveis por 50\% das mortes nos EUA - Estados Unidos da América. (KRUMEL,1998)

A associação das dislipidemias com as doenças cardiovasculares faz com que a abordagem terapêutica seja mais ampla e considere não só o perfil lipídico do paciente, mas também qual a doença cardiopata associada e quais os fatores de risco a ela associado, como: tabagismo, hipertensão arterial sistêmica (HAS), idade, raça, sexo, etilismo,obesidade, sedentarismo, história patológica familiar (HPF). (COTRAN,2000)

Todos os estágios da lesão aterosclerótica são reversíveis, desde que o fator que gera a lesão seja removido e as ações protetoras consigam cessar o quadro inflamatório e fibroproliferativo da lesão. (DEHOOG,1998)

\section{Dislipidemia e Aterosclerose}

O quadro de dislipidemia, o principal fator desencadeador da aterosclerose, é caracterizado como alterações qualitativas e/ou quantitativas dos lipídeos e lipoproteínas sanguíneas, que podem acontecer em decorrência de vários fatores (hábito alimentar, obesidade, sedentarismo, tabagismo, etilismo, distúrbios genéticos); a dieta é o mais importante deles. O acúmulo de uma ou mais classes de lipoproteínas é causado pela deficiência de remoção do plasma ou maior produção. (METZE,2000)(LIBBY,2000)

A Sociedade Brasileira de Cardiologia (SBC) recomenda que a medição dos níveis de colesterol comece aos 30 anos, pois já se sabe que em qualquer idade quanto mais alto o nível de colesterol sanguíneo maior o risco de doenças cardiovasculares; o controle traduz uma evidente redução das cardiopatias. (GEBARA,1999)

Aterosclerose é uma doença de lento desenvolvimento, com início precoce e tem o ateroma, que é o depósito de lipídeos na íntima do vaso como característica. O início do processo aterosclerótico é desencadeado pela oxidação das moléculas de lipídeo que ficam circulantes no plasma, essa oxidação acontece devido à atuação dos radicais livres, moléculas de oxigênio modificadas que vão lesar ou desencadear alterações que vão causar patologias (aneurisma, infarto agudo do miocárdio, gangrena, trombose). Os fatores e ações que desencadeiam a formação excessiva de radicais livres são tabagismo, etilismo, exercício físico, estresse. (PRADO,2001)

É uma lesão de característica silenciosa até que se complique por estenose, trombose, aneurisma ou embolia.(COTRAN,2000) (BERKOW,1989) Segundo a OMS as ateroscleroses coronarianas juntamente com as doenças cerebrovasculares, vão liderar as causas de mortalidade em todo o mundo na próxima década; além disso, a aterosclerose é a doença mais importante do ponto de vista da saúde pública. (ZACHÉ,2005) 
O LDL-colesterol é uma lipoproteína de baixa densidade e o seu excesso na circulação sanguínea lesa os vasos após ser oxidado pelos radicais livres, facilitando o depósito nesses canais e aumentando os riscos de provocar doenças cardiovasculares. Segundo a Sociedade Brasileira de Cardiologia, “o LDL-C é fator causal e independente de aterosclerose e sobre o qual deve-se agir para diminuir a morbi-mortalidade”. (ELIAS,2001)

O LDL transporta os ésteres de colesterol e vão interagir com as células endoteliais, modificadas por algum fator, para entrar no espaço subendotelial. No subendotélio o LDL é oxidado por oxigênios reativos (radicais livres) liberados pelos monócitos, e após ser transformado em LDL-oxidado será incorporado por células de macrófagos (monócitos evoluídos). Os macrófagos incorporam o LDL-oxidado até virarem células espumosas estouram e liberam partes celulares necrosadas e colesterol, que serão depositados na parede endotelial obstruindo progressivamente a luz do vaso. Com o tempo há calcificação da lesão, fissura ou ulceração na placa, hemorragia intravascular, perda da elasticidade vascular pela presença de placa fibrosa. A etiologia é complexa e depende de muitos fatores não totalmente esclarecidos. A afecção atinge praticamente todas as partes do globo, havendo apenas variações de sua intensidade, gravidade e complicações; depósitos lipídicos na íntima arterial começam a aparecer na criança a partir de 1 ano de vida. (METZE,2000)

A prevalência e a gravidade da aterosclerose estão relacionadas com diversos fatores que podem ser modificáveis ou não-modificáveis. Os modificáveis ou potencialmente controláveis são: dislipidemia (HDL baixo e LDL elevado), hipertensão, tabagismo, diabetes, sedentarismo, obesidade. Os não-modificáveis são: idade, sexo, raça, história familiar, anormalidades genéticas. (SANTOS,2005)

Qualquer pessoa pode desenvolver lesão aterosclerótica, mas algumas estão mais predispostas devido à presença de alguns fatores de risco. A presença de múltiplos fatores de risco exerce efeito negativo no desenvolvimento de aterosclerose; dois fatores associados aumentam cerca de quatro vezes o risco, a presença de três fatores faz com que a taxa de infarto do miocárdio seja sete vezes maior do que na ausência destes. (COTRAN,2000)

Os estudos de prevenção das doenças aterosclerosas visam a redução dos custos causados por DCV, pois o ônus tem crescido exponencialmente nas últimas décadas. No ano de 2000 as DCV foram responsáveis pela principal alocação de recursos públicos em hospitalizações no Brasil, cerca de 821 milhões de reais, e foram a terceira causa de permanência hospitalar prolongada. Entre 1991 e 2000 os custos das DCV aumentaram 176\%. As seqüelas clínicas da aterosclerose representam uma cifra expressiva de gastos em saúde, cerca de $\mathrm{R} \$ 51.211$ por paciente com infarto agudo; $\mathrm{R} \$ 24.890$ para angina de peito. É importante enfatizar a escassez de análises econômicas que relacionem a intervenção médica terapêutica e a intervenção preventiva no Brasil.(SANTOS,2005)

\section{Abordagem Terapêutica Convencional}




\section{(medicamentosa e nutricional)}

A principal ação contra as dislipidemias é a dietoterapia, sendo esta uma conduta terapêutica indicada isoladamente ou em conjunto com outros tipos de tratamentos; mas não existe qualquer outro tipo de tratamento das dislipidemias sem estar associado a condutas dietoterápicas. (SANTOS,2005)

A dieta deve incluir redução das gorduras saturadas; redução do colesterol alimentar; redução dos carboidratos simples que elevam os níveis de triglicerídeos devido a metabolização hepática que os transforma em reserva energética; aumento das fibras dietéticas (solúveis e insolúveis) para melhor eliminação das gorduras; aumento de antioxidantes (fitoquímicos, vitaminas, compostos bioativos) para redução dos radicais livres; busca pelo peso ideal para reduzir os efeitos da lipogênese no quadro dislipidêmico.

A terapia medicamentosa é usada para controlar a dislipidemia e reduzir a dor anginosa, por isso são empregadas drogas que reduzem os lipídeos, a agregação plaquetária e a pressão arterial; que controlam o diabetes; diminuem a freqüência cardíaca, a força de contração do miocárdio e/ou dilatam as artérias coronárias. (SOUSA,2005)

Durante a prescrição das drogas são fundamentais as interações com a nutrição para evitar reações adversas (vômitos, náuseas, diarréias) e perda ou excesso de algum nutriente. O tipo de droga é escolhido de acordo com a severidade do quadro dislipidêmicoaterosclerótico do paciente. A principal conduta a ser adotada na prevenção e no tratamento das dislipidemias é a nutricional, que deve ser seguida rigorosamente em conjunto com as mudanças no estilo de vida, que atuam como fatores de risco. (SANTOS,2005)

A dieta deve conter: ingestão mínima de produtos animais com elevado teor de gorduras saturadas, pois contém fatores hipercolesterolêmicos (ácidos graxos, colesterol); elevado percentual de ácidos graxos insaturados (família dos linoléicos - óleos vegetais), produtos marinhos (família dos linolênicos); valores adequados de fibras (frutas, leguminosas, aveia, folhas); mínimo de açúcares simples; aumentar a quantidade de alimentos ricos em substâncias antioxidantes (vitaminas E e C, carotenóides, bioflavonóides). (METZE,2000)

O programa National Cholesterol Education NCEP Adult Treatment Panel III ATP III, recomenda a combinação de terapias alternativas como método eficaz na melhora dos níveis de colesterol em indivíduos com dislipidemia. Dieta pobre em gordura saturada, exercício físico, suplementação nutricional com óleo de peixe, aveia, fitoesteróis. (MAESTÁ,2006)

Segundo a Associação Americana do Coração o tratamento dietético deve seguir as seguintes recomendações: 


\begin{tabular}{|c|c|}
\hline Nutrientes & Ingestão Recomendada \\
\hline Gordura Total & 25 a $35 \%$ das calorias totais \\
\hline Acidos Graxos Saturados & $<7 \%$ das calorias totais \\
\hline Acidos Graxos Polinsaturados & $\mathbf{1 0 \%}$ das calorias totais \\
\hline Acidos Graxos Monoinsaturados & 50 a $60 \%$ das calorias totais \\
\hline Carboidratos & Aproximadamente $15 \%$ das calorias totais \\
\hline Proteinas & $<200 \mathrm{mg} /$ dia \\
\hline Colesterol & 20 a $30 \mathrm{~g} /$ dia \\
\hline Fibras & Para atingir e manter o peso desejável \\
\hline Calorias
\end{tabular}

FONTE:ELIAS,2001.

Quadro 2: Recomendações Dietéticas para o Tratamento das Hipercolesterolemias

As recomendações indicam redução do consumo de gorduras trans, devido a ação dos receptores celulares de LDL-colesterol, que inibem a remoção plasmática e elevam os níveis celulares de LDL; e o aumento no consumo total de gorduras insaturadas, objetivando a redução dos níveis plasmáticos de triglicerídeos e elevação do HDLcolesterol. (ELIAS,2001) (SANTOS,2000)

O interesse no estudo dos ácidos graxos polinsaturados começou à partir da verificação de menor ocorrência de doenças cardiovasculares nos esquimós, pois se verificou que apesar do consumo de uma dieta rica em gorduras eles apresentavam incidências menores de deposição de gordura arterial em relação aos ocidentais; o que pode ser explicado pelo alto consumo de ácidos graxos insaturados. As gorduras insaturadas são ômega-3 (alfa-linolênico, eicosapentaenóico-EPA e docosahexaenoico-DHA), ômega-6 (linoléicos e araquidônico) e ômega-9 (oléico); na dieta típica ocidental é observado maior consumo de ômega-6 em relação a ômega-3, pois estes estão presentes em alimentos como soja, milho, óleo de girassol e açafrão, enquanto ômega-3 é encontrado em peixes de águas frias e profundas (cavala, sardinha, arenque, salmão, truta, bacalhau), óleo de canola, linhaça.(ELIAS,2001) (PIMENTEL,2005)

O consumo excessivo de carboidratos aumenta a síntese de triglicerídeos que vão servir de reserva energética; a lipogênese hepática ocorre a partir de substratos do carboidrato (frutose, galactose) convertendo esses glicídios em TG (precursores de moléculas de colesterol). A ingestão não deve ultrapassar as recomendações da RDA, que é de 50 a $60 \%$ das calorias totais da dieta. (NUNES,2005)

O consumo de fibras (polissacarídeos não amiláceos) é recomendado como agente terapêutico das dislipidemias, por se ligarem aos ácidos biliares aumentando a degradação do colesterol (eliminação fecal) e excreção pela via dos ácidos biliares (síntese de bile).(ELIAS,2001) (JORGE,2001)

É recomendado adaptar gradualmente uma dieta rica em fibras solúveis e insolúveis (20 a 30g/dia), concomitantemente com o aumento do volume de água ingerida durante o 
dia; pois comer alimentos com alto teor de fibras em cada refeição regula a função gastrintestinal além de regular o perfil lipídico.

A cocção reduz o teor de fibras e os alimentos fontes de fibras solúveis são: aveia, farinha de aveia, feijões, ervilhas, frutas cítricas, maçãs, morangos, framboesas; alimentos fontes de fibras insolúveis são: pães integrais, cereais, cenouras, couves, farelos, casca de maçã. (PIMENTEL,2005)

\begin{tabular}{|c|c|c|}
\hline Alimento $(100 \mathrm{~g})$ & Fibra Soluvel & Fibra Insolúvel \\
\hline Alface & 0,57 & 1 \\
\hline Arroz branco* & 1,26 & 1,52 \\
\hline Arroz Integral $\left.\right|^{* *}$ & 0,96 & 3,37 \\
\hline Aveia em flocos & 4,56 & 1,86 \\
\hline Berinjela* & 0,94 & 1,59 \\
\hline Beterraba* & 1,42 & 1,29 \\
\hline Brócolis* & 0,18 & 1,7 \\
\hline Cenoura & 1,63 & 1,6 \\
\hline Chuchük & 0,35 & 0,79 \\
\hline Couwe: & 0,77 & 1,23 \\
\hline Couve-flor* & 1,01 & 1,52 \\
\hline Ervilha* & 2,22 & 1,38 \\
\hline Ervilha em conserva & 1,32 & 3,93 \\
\hline Farelo de aweia & 3,4 & 3,56 \\
\hline Farelo de trigo & 3,41 & 36,04 \\
\hline Feijầo carioca* & 2,6 & 4,62 \\
\hline
\end{tabular}

\begin{tabular}{|c|c|c|}
\hline Alimento $(100 \mathrm{~g})$ & Fibra Solúvel & Fibra Insolúvel \\
\hline Feijão preto* & 1,77 & 3,61 \\
\hline Jiló & 6,84 & 1,82 \\
\hline Lentilha* & 1,81 & 5,44 \\
\hline Macarrão inte gral* & 1,04 & 2,18 \\
\hline Mandioca* & 2,72 & 2,14 \\
\hline Pão de Centeio & 2,32 & 4,83 \\
\hline Pão francês & 2,5 & 3,86 \\
\hline Pão integral & 1,79 & 5,24 \\
\hline Rabanete & 0,86 & 0,74 \\
\hline Repolho & 1,06 & 1,6 \\
\hline Soja & 0,71 & 3,37 \\
\hline Tomate & 0,31 & 0,81 \\
\hline Vagem $^{*}$ & 1,13 & 2,74 \\
\hline
\end{tabular}

FONTE:SALGADO,2000. * * Alimento cozido

Quadro 3: Teor de fibras em alguns alimentos

Lairon et al, concluíram em um estudo com 2532 homens e 3429 mulheres, que o consumo de fibras dietéticas está inversamente relacionado com diversos fatores de risco 
para doenças cardiovasculares em ambos os sexos, ou seja, as fibras têm papel protetor contra doenças cardiovasculares e o aumento do seu consumo deve ser incentivado. (LAIRON,2005)

Os íons de ferro estão envolvidos na formação de radicais livres, por isso existem evidências de que o excesso de ferro pode acelerar o desenvolvimento de aterosclerose; além disso, pode ser que a menor incidência de DCV em mulheres em idade fértil esteja relacionada à perda periódica de ferro pela menstruação (além da função protetora dos estrógenos). Alguns autores avaliam que a doação periódica de sangue pode ter efeito protetor contra as doenças cardiovasculares pela redução de disponibilidade de ferro no organismo.(METZE,2000)

\section{Alimentos Funcionais}

O homem sempre esteve em busca de saúde e longevidade, pois a história nos aponta sua tendência em transformar alimentos em medicamentos. A observação da menor incidência de determinadas doenças em grupos populacionais com dietas diferenciadas, como a asiática (baixo consumo de carnes vermelhas e elevado consumo de soja), a do mediterrâneo (aumenta o consumo de peixes, frutas e vegetais), a dos esquimós (elevado consumo de peixes gordurosos); incentivou inúmeros estudos que visam elucidar os mecanismos de proteção atuantes em cada tipo de dieta. Diferentes alimentos com diferentes atividades biológicas estão sendo estudados, o que segundo a International Life Sciences Institute, criou uma nova ciência denominada "Ciência dos Alimentos Funcionais”.(VIGGIANO,2005)

Conhecidos há milhares de anos na cultura popular, somente no século XX os compostos ativos dos alimentos começaram a ser estudados de modo objetivo quanto às propriedades fisiológicas e medicinais, como: antioxidantes, diminuição de agregação plaquetária, alteração no metabolismo do colesterol, redução da pressão sanguínea, controle do metabolismo endócrino, detoxificação de carcinógenos. (FERRARI,2005) Há 2500 anos Hipócrates afirmava que a alimentação é responsável pela manutenção da saúde e prevenção de doenças, dizendo “Faz do alimento o teu medicamento”.(OLIVEIRA, 2002) (SILVA,2005) (SALGADO,2004)

Alimento natural com propriedades funcionais deve ter consumo regular e uma preparação adequada para atingir suas funcionalidades. É indispensável cautela científica nas aprovações legais de propriedades funcionais de alimentos; por isso a Agência Nacional de Vigilância Sanitária (ANVISA), vinculada ao Ministério da Saúde (MS) publicou

portarias e resoluções que definem a legislação de alimentos funcionais. (OLIVEIRA,2006)

Os alimentos funcionais também podem ser chamados de: nutracêuticos, nutragênicos, medical food, nutritional food, pharmafood, therapeutic food, fitness food, biocêuticos. Eles apresentam substâncias com funções biológicas distintas, denominadas de compostos bioativos responsáveis por garantir a manutenção da saúde. (FARRET,2005) 
Os principais alimentos funcionais e compostos bioativos que previnem as doenças cardiovasculares são: alho (alicina e quercetina), cebola (quercetina), tomate (licopeno), berinjela (cinarina e ácido caféico), chocolate amargo (epicatequina, catequina e procianidinas), uva e vinho tinto (resveratrol, luteonina, quercitina, procianidinas e taninos), peixe (ômega-3), oleaginosas (fitoesteróis), chá verde (catequinas), soja (isoflavonas, daidzeína e genisteína), maçã (cianidina, quercetina, catequina), especiarias (ácido rosmarínico, carnosol, ácido carnosónico, rosmarifenol, rosmariquinona), cogumelos (eritadenine), leguminosas (isoflavonas, saponinas, betaglucanas). (PASCHOAL,2001)

A nutrição funcional deve considerar a individualidade bioquímica, o equilíbrio nutricional, a biodisponibilidade de nutrientes; pois esta é uma atuação cientifica e integrativa que trata de doenças e promove o bem-estar entre todos os sistemas do corpo enfatizando a fisiologia. (PASCHOAL,2006)

As principais ações dos compostos ativos dos alimentos funcionais na prevenção da aterosclerose englobam o poder antioxidante, inibição da agregação plaquetária e seu efeito hipotensor.

\section{- Antioxidantes}

São compostos alimentares que removem radicais livres e espécies reativas do oxigênio, nitrogênio e cloro, conhecidamente tóxicos para as células. Esses elementos tóxicos são liberados na respiração mitocondrial, durante atividades normais. Exercícios físicos em excesso, diversos estados fisiopatológicos (infecções, doenças cardiovasculares, câncer, diabetes e estresse) geram um aumento da produção dos radicais livres e suas reações geram conseqüências como: deterioração de lipídeos, aterosclerose e doenças cardiovasculares, diabetes, perda de nutrientes e morte celular. (FERRARI,2005) (ZBORAJ,2006)

Esses compostos antioxidantes protegem células, tecidos e vários órgãos que são vitais. Destacam-se os seguintes antioxidantes: vitaminas A, C, E e ácido fólico; flavonóides (soja, chá verde, cebola, alho, maçã); antocianinas (feijão, uvas, morango, cereja); carotenóides (cenoura, abóbora, tomate, goiaba); ervas e condimentos (alecrim, sálvia, tomilho, orégano). (ZBORAJ,2006)

As vitaminas A, C E e ácido fólico têm efeito importante na redução da oxidação das lipoproteínas, impedindo a formação dos radicais livres, são encontrados em frutas cítricas, 
vegetais verdes, nozes, uva, abacate, grãos, leite, leguminosas. O ácido fólico também atua na redução dos níveis plasmáticos de homocisteína, que como visto é um aminoácido que atua como agente aterogênico. (JUZWIAK, 2004.)

Segundo Accioly, estudos ressaltam a importância da ingestão de frutas e vegetais, pois neles são encontrados potentes antioxidantes; ela afirma que um conjunto de medidas dietéticas aliadas a mudanças no estilo de vida, pode contribuir para a redução dos casos de mortes por DCV e suas seqüelas.(ACCIOLY,2002) A utilização freqüente e prolongada de alimentos que desempenhem propriedades antioxidantes, torna-se muito importante na prevenção de doenças crônicas. (OLIVEIRA, 2002)

\section{- Inibição da agregação plaquetária}

A agregação plaquetária é um estágio crítico durante a progressão da placa de ateroma e na formação de trombos, os principais fatores que levam a formação dessas agregações são: tabaco, estresse, níveis elevados de colesterol e lipoproteínas, homocisteinemia, diabetes, além disso há diminuição de síntese de óxido nítrico e prostaciclinas pelo endotélio que reveste as placas de ateroma, componentes com importante ação inibitória de agregação plaquetária. (STUMP,1998) (BRASILEIRO FILHO,2000) (LIBBY,2000)

Os compostos bioativos diminuem a agregação plaquetária, principalmente os polifenóis do chá, uva, vinho tinto, cacau, com potente ação de inibição da agregação plaquetária, reduzindo a formação de trombos que obstruem o fluxo sanguíneo, podendo provocar a formação de placas ateroscleróticas, isquemia e hipóxia (falta de oxigênio tecidual). (FERRARI,2005)

\section{- Redução do colesterol}

As alterações no metabolismo do colesterol são evidenciadas à partir da diminuição da produção hepática ou diminuição da absorção intestinal. O controle do metabolismo endócrino é baseado no consumo de flavonóides capazes de modular o metabolismo de colesterol e diminuir o risco de doenças cardiovasculares. (CARDOSO,2005) 


\section{- Hipotensor}

A redução da pressão arterial através de uma dieta com efeitos hipotensores (rica em frutas, verduras e teor reduzido de sal), aliada a prática de exercícios físicas e obtenção de peso adequado, são condutas fundamentais para a manutenção e normalização da pressão arterial.

Os principais agentes hipotensores são os polifenóis (chá, vinho, uva) e a arginina (oleaginosas), que têm a capacidade de elevar a produção de óxido nítrico (vasodilatador) e inibir a endotelina-1, fator que promove a contração endotelial e aumenta a pressão.(FERRARI,2005)

Os compostos ativos responsáveis pelas ações bioquímicas preventivas das doenças cardiovasculares (redução do perfil lipídico, redução da agregação plaquetária, hipotensão) pertencem ao grupo dos polifenós (flavonóides e isoflavonas), carotenóides e ômega - 3. (AÇÃO COST,2001)

\section{- Polifenóis - flavonóides e isoflavonas:}

Os polifenóis são uma classe de compostos com mais de 8000 representantes, que são responsáveis pela pigmentação, pelo crescimento, pela reprodução, pela resistência a patógenos e por muitas outras funções nas plantas. Os polifenóis aqui discutidos são flavonóides (flavonas/flavononas, antocianinas, catequinas) e isoflavonas (fitoestrógenos).

Os flavonóides são divididos em grupos: flavonas, flavanonas, antocianinas e catequinas; sendo encontrados em frutas (maçã, uva, amora, framboesa, frutas cítricas), hortaliças (cebola, alho), chá, vinho, chocolate, pimenta, leguminosas. (AÇÃO COST,2001) (OLIVEIRA - 2002)

Após sofrerem metabolização no intestino delgado, os flavonóides atuam como antioxidantes, através da alteração da produção de radicais livres, eliminação de precursores dos radicais livres, quelação de metais e elevação de antioxidantes endógenos. Acredita-se que a ingestão regular de alimentos fontes desses compostos auxilie a prevenção das doenças cardiovasculares, pois regulam a permeabilidade capilar permitindo o fluxo constante de oxigênio e nutrientes essenciais; além de relaxarem os músculos do sistema cardiovascular através de ações hipotensoras; evitam a formação de coágulos; previnem a oxidação de LDL por radicais livres. (PIMENTEL,2005)

As isoflavonas e lignanas também formam uma subclasse dos polifenóis, mas são discutidas como fitoestrógenos pois têm uma estrutura química comparada a um hormônio humano conhecido como estradiol; o que fez dos alimentos representantes desse grupo famosos por estarem associados a uma forma de terapia natural de reposição hormonal. 
Existem mais de 800 vegetais com fitoestrógenos em sua composição, mas a soja e o cravo são os principais. (AÇÃO COST,2001) Por serem polifenóis têm As mesmas funções que os flavonóides e outras específicas; dentre as ações preventivas das doenças cardiovasculares reduzem as concentrações de colesterol e LDL-colesterol, além de terem efeitos antioxidantes. (PIMENTEL,2005)

\section{- Carotenóides:}

Os carotenóides são substâncias lipossolúveis que estão em abundância nos alimentos (mais de 400 tipos), mas apenas uma pequena parcela desses compostos são consumidas em quantidades significativas para que os efeitos preventivos aconteçam. As principais fontes de carotenóides são: cenoura, abóbora, manga, mamão, tomate, goiaba, melancia, dentre muitos outros. Por serem hidrofóbicos, a absorção é facilitada pela presença de lipídeos da dieta e enzimas digestivas (lípases); e a presença de fibras solúveis pode reduzir a absorção dos carotenóides. O principal carotenóide e que terá maior enfoque no trabalho é o licopeno com potente ação antioxidante e mais biodisponíveis após a cocção; os representantes desse composto são: tomate, goiaba, melancia. (PIMENTEL,2005)

\section{- Ômega-3:}

Ômega-3 são ácidos graxos capazes de provocar alterações na função plaquetária, na resposta imune (atuando como se fossem ácido araquidônico), têm ação vasodilatadora e anti-agregante plaquetária, redução de triglicerídeo plasmático devido inibição da secreção hepática de VLDL; fatores esses que contribuem para a prevenção de doenças cardiovasculares. As principais fontes são peixes de águas frias e profundas; óleos de linhaça, de canola e de gérmem de trigo.(PIMENTEL,2005)

Os ácidos graxos ômega - 3 e 6 são precursores de eicosanóides, substâncias que ajudam a regulação da pressão arterial, vasodilatação, lipólise e resposta imune.(PASCHOAL,2001)

Os alimentos funcionais que serão elucidados abaixo com ação preventiva e de controle das doenças cardiovasculares foram preteridos, pois são alimentos usuais, são eles: alho, tomate, berinjela, chocolate amargo, uva ou vinho tinto, peixe, oleaginosas (nozes, castanha-do-pará, avelã), chá verde, soja e derivados, especiarias, leguminosas, cogumelos e maçã.

\section{- Alho}

O alho pertence à família Liliacerae com aproximadamente 88 espécies de alho em todo o mundo. O alho é um tempero que tem fama de “curador”, pois atribuem a ele a capacidade de interferir numa série de doenças; no Egito o alho era usado para remediar a diarréia e na Grécia antiga para o tratamento de patologias pulmonares e intestinais; e pesquisas recentes comprovaram que dentre suas atividades as que mais se destacam são as antimicrobianas, antineoplásicas, antiaterogênicas, imunoestimulatórias e hipoglicemiante, hipotensor, antiviral, antifúngico, hipocolesterolemico. O composto ativo mais estudado atualmente é a alicina, agente antibacteriano formado no alho à partir do seu corte ou 
trituração,levando a atuação da alinase na conversão da aliina em alicina. Os compostos ativos são sensíveis ao calor e devem ser acrescidos ao alimento pronto; evitando a exposição ao fogo.(QUINTAES,2006) (PASCHOAL,2001)

Desde a década de 80, vários estudos utilizando o alho in natura ou em diferentes preparações foram executados buscando seus efeitos na prevenção de doenças cardiovasculares, redução das concentrações séricas de LDL, triglicerídeos, hipotensor e inibidor da agregação plaquetária. (CARDOSO,2005)

Quintaes, alega que o consumo regular de no mínimo 8g/dia, reduz o nível do colesterol sérico total, evita a agregação plaquetária e atua como antioxidante combatendo os radicais livres; que se deve à inibição de diversas enzimas da síntese hepática de colesterol e à um acréscimo na excreção de ácido biliar. (QUINTAES,2006)

ADA (American Dietetic Association), sugere o consumo de 600 a $900 \mathrm{mg}$ por dia, o que representa aproximadamente 1 dente de alho fresco. (2001) Segundo Campbell, o alho é um agente antiaterogênico pois inibe a proliferação de células musculares lisas e reduz a concentração sérica de lipídeos. (2001)

\section{- Cebola}

A cebola também pertence à família das Liláceas e é considerada uma das principais fontes de flavonóides (principal é a quercetina) e compostos sulfurados da alimentação. O consumo de cebola atua na redução da pressão arterial (hipotensor), da viscosidade plasmática, inibe a agregação plaquetária e previne câncer de estômago. (WILLIAM, 2001) (CHEN,1999)

Diferentes métodos de cocção foram avaliados, sendo a cocção em microondas a que mais reteve substâncias antioxidantes, seguida pela fritura e pela cocção em água (a qual teve redução de 30\% dos compostos). (PASCHOAL,2001). (CARDOSO, 2005)

Kalus et al. demonstraram os efeitos positivos da cebola na redução da pressão arterial, da viscosidade sanguínea e do hematócrito de pessoas que consomem a dieta do mediterrâneo. (2001)

\section{- Tomate}

A principal substância presente no tomate é o licopeno, um carotenóide responsável pela pigmentação vermelha dos alimentos e por reduzir metade dos casos de câncer de próstata; funciona como antioxidante e age na neutralização de radicais livres, protegendo o envelhecimento das células e estimulando o sistema imunológico. (RISSANEN,2003)

A absorção do licopeno pelo organismo é maior quando em molhos ou concentrados, do que em tomates frescos. O processamento térmico induz a isomerização do licopeno que de trans vira cis, melhorando a biodisponibilidade; evitar temperatura superior à $120^{\circ} \mathrm{C}$, pois causa redução na quantidade de licopeno. A presença de lipídeo (preferencialmente extra-virgem por ser gordura insaturada) aumenta a biodisponibilidade de licopeno e sua atividade anti-oxidante plasmática. (JUZWIAK,2004) (PIMENTEL,2005) 


\begin{tabular}{|l|l|}
\hline Alimento & Licopeno $(\mathbf{m c g} / \mathbf{1 0 0 g})$ \\
\hline Ketchup & $99,0-134,4$ \\
\hline Suco de tomate & $50,0-116,0$ \\
\hline Massa de tomate & $54,0-1500,0$ \\
\hline Molho de tomate & 62,0 \\
\hline Mamão papaia & $20,0-53,0$ \\
\hline Goiaba vermelha & 54,0 \\
\hline Melancia & $23,0-72,0$ \\
\hline Tomate fresco & $8,8-42,0$ \\
\hline
\end{tabular}

FONTE:JUZWIAK, 2004.

Quadro 4: Conteúdo de Licopeno em alguns alimentos

Djuric \& Powell et al, avaliaram a capacidade antioxidante de várias preparações a base de tomate, concluíram que a sopa de tomate foi a com melhor potencial antioxidante, mas alegaram que o consumo de uma variedade de alimentos a base de tomate é fundamental para a obtenção de uma melhor atividade antioxidante provinda da dieta.(2001)

Segundo alguns estudos o consumo de 1 tomate ou 1 colher de sopa de massa de tomate por dia é responsável pela inibição de cerca de 93\% da formação de peróxidos lipídicos, pois o licopeno age em conjunto com outros antioxidantes na oxidação do LDL garantindo uma maior característica antiaterogênica. (PASCHOAL,2001)

Rissanen et al, verificaram que o consumo e a concentração sérica de licopeno pode ter importância clínica e de saúde pública, pois concluíram que o licopeno tem papel importante no combate do estágio inicial da lesão aterosclerótica. (2003)

\section{- Berinjela}

Possui nome científico Solanum melongena L., é originária da África e é um alimento que ajuda no controle dos níveis de colesterol e triglicerídeos. A cinarina e o ácido caféico são os compostos bioativos da berinjela responsáveis pela redução dos níveis sanguíneos de colesterol, pois estimulam a produção da bile hepática. A berinjela pode ser usada na forma in natura liquefeita, tintura, extrato. (GONÇALVES,2004) (GUIMARÃES,2000) (JORGE,2001)

Gonçalves, verificou em vários estudos farmacológicos que a berinjela tem sido usada no combate de hipercolesterolemia, angina, infarto do miocárdio, acidente vascular e algumas patologias renais e hepáticas. O chá e o suco de berinjela atuam como coadjuvante das dietas pois aumentam a eliminação de gorduras, segundo Guimarães et al., o chá com $10 \mathrm{~g}$ de berinjela durante 5 semanas tem efeito modesto na diminuição de hipercolesterolemia devido excreção fecal de ácidos biliares. (GONÇALVES,2004)

Contradizendo, Praça et al., realizaram um estudo e verificaram que o suco de berinjela com laranja não tem efeito hipocolesterolêmico a ponto de substituir o tratamento 
com estatinas, por isso não deve ser usada como uma intervenção terapêutica alternativa.(2004)

Guimarães e cols., verificaram efeitos transitório e modesto da berinjela em indivíduos hipercolesterolêmicos, efeitos esses observados em pacientes sob orientação da dieta hipocolesterolêmica; o autor justifica essa atuação singela relacionando os níveis de compostos ativos, pois verificou em outros estudos que os concentrados de berinjela (extratos, tinturas) exercem melhores efeitos. (PRAÇA,2004)

\section{- Chocolate Amargo}

Entre o século XVI e XX foram encontradas mais de 100 indicações sobre o uso do chocolate com fim medicinal. O chocolate é produzido à partir da incorporação da massa de cacau de sacarose, manteiga de cacau, substâncias flavorizantes, leite, amêndoas, passas.(GOLLUCKE,2004)

É um produto com alto teor de gordura: 35\% de ácido oléico (gordura monoinsturada), 35\% ácido esteárico e 25\% ácido palmítico (gordura saturada) e 5\% outros. O ácido esteárico apesar de saturado não eleva os níveis de colesterol no sangue pois parte dele é convertido à acido oléico que reduz levemente o colesterol; já o ácido palmítico tem efeito hipercolesterolêmico. (GOLLUCKE,2004)

Contém epicatequina, catequina (monoméricas) e procianidinas (oligoméricas), que são flavonóides que têm ação antioxidante e protegem o organismo dos danos oxidativos causados pelo acúmulo de LDL colesterol oxidado pelos radicais livres, reduzindo o risco de doenças cardíacas. (WOLLGAST,2000) (WAN,2001) (MELZIG)

Segundo Osakabe, o consumo de 36 gramas de cacau em pó por dia é capaz de atuar como um antioxidante.(PASCHOAL,2001)

Como mostra a tabela abaixo, o teor de polifenóis no chocolate amargo é maior do que no chocolate ao leite, por isso e devido a quantidade de açúcar, o consumo do chocolate ao leite não é tão estimulado quanto o amargo.

\section{- Uva e Vinho Tinto}

Existem hoje mais de 250 mil trabalhos de pesquisa que estudam os efeitos do vinha na saúde; a Sociedade Brasileira de Cardiologia reconhece a ação antioxidante e preventiva do vinho. Sabe-se que existem mais de 1000 substâncias ativas e que 600 delas já foram estudadas; mas é indiscutível que o dano orgânico ocasionado pela ingestão elevada de álcool ocorre quando esse consumo é maior do que a capacidade do organismo em metabolizá-lo. (SOUZA FILHO,2006)

As uvas e sucos de uvas (inclusive os comerciais), são ótimas fontes naturais de antioxidante, o principal é o vinho tinto, pelo seu alto teor de polifenóis e compostos fenólicos, potentes antioxidantes. O composto que mais se destaca é o resveratrol, localizado na casca das uvas, principalmente em maior quantidade nas uvas vermelho-roxas escuras, por isso o vinho tinto tem mais resveratrol que vinhos brancos e rosés (durante a fermentação dos vinhos tintos, as cascas das uvas são incorporadas). (ISHIMOTO,2004) 
Além do resveratrol, a luteonina, a quercetina, as procianidinas (polpa das uvas) e os taninos (casca das uvas), são substâncias naturais encontradas nas uvas com ação antioxidante, inibidores da oxidação lipídica, inibidores da agregação plaquetária, com atividade antiinflamatória; além de aumentarem a resistência das fibras colágenas e impedirem a destruição de linfócitos de defesa, preservando assim o sistema imunológico. (ISHIMOTO,2005)

Cientificamente, sabe-se que é possível associar o consumo de vinho com benefícios à saúde, mas isso só acontece se ele for consumido com moderação e regularmente, junto com as refeições e por pessoas que não apresentem contra indicação. (SOUZA FILHO,2006) (LOPES,2005)

\section{- Leguminosas}

É um importante grupo alimentar formado por sementes contidas em favas como o feijão, ervilha seca, lentilha, grão de bico e soja. Sabe-se que dietas contendo leguminosas apresentam efeitos hipocolesterolêmicos por meio da redução de LDL-colesterol, efeito esse decorrente da ação de substâncias como isoflavonas, saponinas e beta-glucanas. A soja é o alimento de maior enfoque deste grupo, por isso será elucidada em tópico à parte.(PASCHOAL,2001)

São encontrados compostos fenólicos nas leguminosas, sendo os principais a genisteína, daidzeína, genistina e daidzina. As leguminosas englobam um grupo rico em nutrientes e estão sendo abertos novos horizontes para esses alimentos, e o consumo diário está sendo incentivado pela Organização Mundial de Saúde. Ainda são necessárias pesquisas que definam doses farmacêuticas exatas, mas atualmente observa-se que meia xícara de feijão cozido por dia produz efeitos benéficos à saúde. (ELIAS,2005)

\section{- Soja}

Há alguns anos o estudo em relação aos benefícios da soja à saúde humana começou a ser ampliado, considerando não só sua alta qualidade protéica, mas também seu papel preventivo e terapêutico de doenças como as cardiovasculares, câncer, osteoporose.(SALGADO, 2004)

Pertence ao grupo das leguminosas e apresenta características que devem ser destacadas dentre os alimentos com características funcionais. Os principais fitoquímicos são as isoflavonas (daidzeína e genisteína). (PASCHOAL,2001)

Os produtos à base de soja se relacionam com a prevenção da aterosclerose pois têm ação antioxidante (isoflavonas) sobre as gorduras circulantes no plasma e também pela presença de fibras solúveis que atuam na redução de colesterol e LDL; (MARCÍLIO, 2006) além de exercer efeito positivo na agregação plaquetária, atuar como antioxidantes, diminuir os níveis TG e elevar o HDL. (PASCHOAL, 2001) (ANTHONY,1998) 
O consumo de 25 gramas de proteína de soja por dia vai ajudar na promoção desses efeitos mencionados.(PASCHOAL,2001) (CARDOSO,2005)

Pimentel verificou no estudo de Anderson e cols, que o consumo de 30 gramas de proteína de soja por dia produz efeitos positivos à saúde;(2005) em 100 gramas de grãos de soja tem 37 gramas de proteína de soja. (PASCHOAL,2001)

\section{- Chá-verde}

As propriedades do chá são conhecidas há mais de 4000 anos, feito da Camellia sinensis é uma das bebidas mais consumidas no mundo. Pode ser encontrados como chá verde, chá Oolong e chá preto, mas o método de transformação promove alterações que fazem do chá verde o menos afetado em relação ao Oolong e preto, respectivamente, pois tem maior concentração de compostos fenólicos (quercetina, caempferol, miricetina, apigenina e luteolina) e catequinas (epicatequina, e epigalocatequina) que vão atuar na prevenção de doenças crônico-degenerativas, devido potente ação antioxidante que limita a peroxidação lipídica do LDL, reduz os níveis plasmáticos de colesterol e triglicerídeos. (JUZWIAK,2004) (CARDOSO,2005) (HONG,2000) (CRESPY,2005)

O chá verde é processado através de vaporização ou secagem em temperaturas elevadas com cuidados para prevenir a oxidação dos compostos fenólicos; o chá Oolong e aquecido por ar durante 1 a 2 horas, processo que retém quantidades consideráveis de catequinas,sendo considerada uma opção intermediária entre chá verde e preto; o chá preto é 78\% do chá consumido no mundo e as folhas são mantidas quentes por cerca de 6 horas, provocando perda de grande parte dos compostos fenólicos e catequinas. (PASCHOAL,2001) (KUO,2005)

Em relação às doenças cardiovasculares o chá verde atua na diminuição dos níveis de colesterol, hipotensor, antioxidante e inibidor da agregação plaquetária; mas também tem efeito benéfico como anticancerígeno, regulam a glicose sanguínea, tem propriedades termogênicas que auxiliam na perda de peso. (PASCHOAL,2001)

O preparo deve ser feito cuidadosamente considerando a quantidade e o tempo de infusão, ou seja, 1 colher rasa de que do chá verde para cada xícara de água fervendo, deixando-se em infusão por quatro a oito minutos (tempo para os princípios ativos passarem para a água). O consumo diário de 5 a 7 xícaras de chá promove efeitos terapêuticos, ressaltando que o aquecimento pode levar a uma diminuição da biodisponibilidade das catequinas.(SALGADO,2004)

Segundo Lopes, um estudo recente descobriu que o chá previne a aterosclerose, evitando a formação de placas calcificadas na aorta através da inibição da oxidação do LDL.(2005)

\section{- Especiarias}

Algumas ervas e condimentos apresentam compostos ativos que auxiliam no combate e na prevenção de alterações fisiológicas que podem contribuir para o desenvolvimento da 
aterosclerose. A sálvia possui luteolina e campferol; o alecrim tem carnosol e ácido carnosóico (responsáveis por 90\% das propriedades antioxidantes deste condimento); a pimenta possui carotenóides. (PASCHOAL,2001)

A ação antioxidante das especiarias e ervas tem sido evidenciada pela presença de compostos fenólicos que reduzem a oxidação lipídica devido inibição dos radicais livres; as principais são: cravo-da-índia, sálvia, alecrim, pimenta, tomilho, orégano. Segundo Moreira, a ação preventiva das substâncias fenólicas presentes nessas especiarias atua sobre a oxidação lipídica.(MOREIRA,2004)

Moreira verificou em alguns estudos experimentais com especiarias os benefícios e os compostos ativos destes condimentos, comprovando a ação preventiva e terapêutica como agentes antioxidantes; as quantidades para que os efeitos positivos sejam evidenciados não foram definidas, são necessários mais estudos em torno desse grupo. (2004)

\section{- Cogumelos}

Cogumelos são fungos que tiveram registro a cerca de 3000 a 7000 anos atrás, atualmente o interesse e a utilização dos cogumelos comestíveis tem aumentado dia após dia, devido seu sabor na culinária e seu valor nutricional. (ABACKERLI,2005)

Existem muitas espécies de cogumelos, no Brasil os mais consumidos que apresentam propriedades medicinais devido presença do composto ativo eritadenine, são os Agaricus blazei (“cogumelo do sol”) e o Lentinus edodes (shiitake); cogumelos com evidências científicas de modulação no sistema imunológico, anticolesterolêmico, antiinflamatório, hipotensor.As quantidades e modo de preparo para melhor biodisponibilidade dos compostos ativos ainda não foram definidos, sendo necessário mais estudos.(MARTENSEN,2005)

Especialistas estimam que existam cerca de 10 mil espécies de cogumelos, e está sendo investigado o uso potencial como agentes hipocolesterolêmicos.(PASCHOAL,2001)

Muitos estudos foram realizados em torno das substâncias bioativas dos cogumelos que comprovaram a ação terapêutica dos cogumelos reforçando a importância deste alimento como funcional e servindo como base ao nutricionista clínico funcional a estimular e orientar o consumo pela população brasileira.(MARTENSEN,2005)

\section{- Maçã}

A presença de fibras, vitamina C e compostos bioativos (quercetina, cianidina, quercetina) permitem que a maçã exerça funções como regulação do sistema nervoso, proteção de pele e mucosas, proteção do aparelho digestivo e queda de cabelo, reduz formação de coágulos sanguíneos, combate à obesidade, o reumatismo, a gota, tem ação antiinflamatória, é antioxidante, anticancerígena, cardioprotetora, reduz os níveis de colesterol sanguíneo; para tais efeitos recomenda-se o consumo de 1 maçã por dia. (GONZÁLEZ, 1998) (VIDAL,2005) (CURTI,2005) 
Segundo Leontowiz et al. num estudo com ratos, o colesterol hepático teve uma redução o que indica que a maçã impede o aumento do colesterol hepático por inibir a síntese ou por impedir a absorção do colesterol. Curti e Salgado estudaram os efeitos benéficos da maçã, verificando que há uma evidência epidemiológica entre o consumo de alimentos ricos em fitoquímicos e a redução do risco de desenvolvimento de doenças crônicas. (2005)

\section{- Peixe}

Os peixes e seus produtos são excelentes fontes nutricionais, pois têm proteína de boa qualidade e são ricos em minerais.(SALGADO,2005) São classificados como alimento funcional devido à alta concentração de ômega-3 em algumas espécies como o arenque, atum, salmão, sardinha, cavala e truta. O ácido linoléico reduz os níveis de triglicerídeos plasmáticos, colesterol LDL e VLDL, além de atuarem preventivamente a doenças cardiovasculares; pois a síntese de ácido biliar é aumentada pela gordura do peixe.(CARDOSO,2005) (PASCHOAL, 2001) (ROMIJN,2006)

Ômega-3 são ácidos graxos encontrados em peixes gordurosos que vivem em águas profundas, que quando consumidos regularmente reduzem moderadamente os níveis de TG, de agregação plaquetária e pressão arterial.(MARCÍLIO,2006) (LOPES,2005) (ROMIJN,2006)

O consumo de mais de $180 \mathrm{~g}$ de peixe por semana reduz o risco de doença coronariana. As recomendações de ômega-3 das DRIs (Dietary Reference Intakes) giram em torno de um consumo de 0,6 a 1,2\% do valor calórico total, sendo usada uma proporção de ômega-6 : ômega-3 de 2:1 a 4:1, para que os efeitos benéficos dos óleos insaturados seja alcançada. (PIMENTEL,2005)

Taylor et al., concluíram que o consumo de 2 a 4 porções de 180 gramas de peixe por semana é capaz de prevenir doenças do coração, pois o ômega-3 atua como agente protetor.(2006)

\section{- Oleaginosas}

As oleaginosas são a segunda fonte mais comum de resveratrol, além de serem ricas em vitamina E, selênio, manganês e magnésio, micronutrientes responsáveis pela prevenção de DCV através da redução da oxidação de LDL-colesterol. O resveratrol além de ser um antioxidante, também inibe a agregação plaquetária. (MUKUDDEM,2005) (PASCHOAL,2006)

Fonte de arginina, aminoácido capaz de prevenir doenças cardiovasculares através da redução da agregação plaquetária e da dilatação dos vasos sanguíneos pela liberação de óxido nítrico; as oleaginosas também têm efeito hipocolesterolêmico por inibirem a absorção dietética e biliar de colesterol. (PASCHOAL,2001)

Os alimentos que fazem parte desse grupo são: amêndoas, macadâmias, pecãs, avelãs, nozes, castanhas. De acordo com Bricarello, cinco pesquisas clínicas mostraram a relação 
entre as oleaginosas e a doenças coronariana, todas com efeito protetor através da redução significativa de colesterol total e LDL-colesterol.(BRICARELLO,2001)(LOPES,2005)

\section{Conclusão}

A evolução do mundo moderno promoveu profundas mudanças em nossos hábitos; mas nosso organismo ainda está em evolução e não conseguiu se adaptar de forma eficiente a esses novos hábitos, ficando em sobrecarga. A Organização Mundial da Saúde (OMS) estima para 2020 um aumento de até 28\% das mortes relacionadas a problemas cardiovasculares. Um em cada cinco brasileiros apresenta nível de colesterol considerado perigoso pelas entidades internacionais de saúde, totalizando 39 milhões de pessoas, um dos maiores índices do mundo. (ISHIMOTO,2005)

O Consumo regular de alimentos funcionais pode oferecer uma alternativa para reduzir o risco de desenvolver doença coronariana. Benefícios isolados, ou mesmo associados a outros alimentos e/ou terapias que reduzem o colesterol, são opções para a elaboração de cardápios mais saborosos, atrativos, menos restritivos, que promoverá maior adesão à terapia nutricional proposta ao paciente. Atualmente, a ANVISA atesta a funcionalidade de aproximadamente 200 produtos, permitindo a variedade alimentar. (GÓES,2006)

No Japão, Estados Unidos e na Europa, o mercado de alimentos funcionais industrializados já movimenta quase USS 100 bilhões ao ano; no Brasil, apesar de ainda não haver um levantamento preciso sobre a evolução do setor o mercado dá provas de força, pois os consumidores confiam cada vez mais nos efeitos saudáveis de certas substâncias contidas nos alimentos e acreditam que a alimentação é um fator crítico para o controle da saúde.(BRANDÃO,2005)

O paradigma da filosofia da nutrição funcional é a redução do risco de ocorrência de doenças da velhice, mantendo a qualidade de vida. O efeito de muitos alimentos funcionais na prevenção das doenças cardíacas já está devidamente comprovado, embora ainda sejam necessários novos estudos para a definição das quantidades e freqüência a serem consumidas para se obter efeito esperado.

Se consumidos regularmente, associados a hábitos de vida saudáveis e a uma alimentação balanceada, os funcionais podem ser importantes auxiliares na manutenção da boa saúde; é necessário ter em mente que os alimentos funcionais não são milagrosos, por isso é desejável a interação entre médicos e nutricionistas para que essa abordagem resulte em sucesso.

Apesar das contradições em estudos e da ausência de informações mais precisas, verifica-se que os alimentos funcionais são eficazes na prevenção e no controle de algumas doenças, porém a realização de novas pesquisas e determinação de valores que garantam a ação benéfica dos compostos ativos são necessárias. É necessário que a sociedade se conscientize de que as substâncias com atividades funcionais devem ser exaustivamente 
estudadas para serem então recomendados como elementos da promoção da saúde e da prevenção e cura de enfermidades.

Não há mais retorno hoje os componentes alimentares com atividade funcional fazem parte da nutrição aplicada no dia-a-dia de muitos nutricionistas nas suas mais diversas atividades, exigindo desse profissional um aprofundamento na ciência dos alimentos funcionais e a inclusão desta ciência nos currículos dos cursos de graduação e pósgraduação em nutrição.

\section{Referências}

ABACKERLI, Denise. Cogumelos: um alimento funcional milenar redescoberto pela moderna biotecnologia. Revista Nutrição Saúde e performance- Anuário de Nutrição e Alimentos Funcionais.a.6.ed.26.trimestral.abr-jun.São Paulo, 2005.p.12-15.

AÇÃO COST 916 da União Européia.Componentes bioativos dos alimentos vegetais.2001.

ACCIOLY, Elizabeth et.al. Efeito antioxidante das vitaminas A,C,E e aterogênese.Revista Nutrição Brasil.bimestral.jul-ago.2002.

ANTHONY,Mary S.;CLARKSON,Thomas B.;WILLIAMS,J. Koudy.Effects of soy isoflavonas on atherosclerosis: potential mechanisms. American Society for Clinical Nutrition.USA, 1998.

BERKOW, Robert M.D. Manual Merck de Medicina: diagnóstico e tratamento.ed.15.São Paulo:Roca,1989.

BRANDÃO,Sebastião César Cardoso.Novas gerações de produtos lácteos funcionais. Disponível em <www.revistalaticionios.com.br/main_frame/revista/ ed_37pdjs> Acesso em 05 jun. 2005.

BRASILEIRO FILHO,Geraldo.Bogliolo Patologia.Rio de Janeiro:Guanabara Koogan,2000.

BRICARELLO, Liliana Paula; COSTA, Rosana Perim. Nutrição nas Dislipidemias Atualização. Revista Nutrição Saúde e performance: Anuário de Nutrição Clínica.trimetral. 2001.p.14 -17.

BRICARELLO,Liliana Paula.Gordura Monoinsaturada: a mais recomendada. Sociedade Braisleira de Cadiologia.São Paulo, 2005. 
CAMPBELL,Julie H.;EFENDY,Johnny L;SMITH,Nicole J;CAMPBELL, Gordon R. Recent Advances on the Nutritional effects Associated with the use of Garlic as a Supplement.American Society for Nutritional Sciencis.Australia,2001.

CARDOSO, Lúcia Moura.Alimentos Funcionais: o remédio de cada dia. Sociedade de Análise Existencial e Psicomaiêutica Rio de Janeiro,2005.

CHEN,Jia-Huey et al.Chronic Consumption of Raw But Not Boiled Welsh Onion Juice Inhibits Rat Platelet Function . September. Coréia do Sul.1999.

COTRAN, Ramzi S.; SCHOEN, Frederick J.. Vasos Sanguíneos. In: COTRAN, Ramzi S.; KUMAR, Vinay; COLLINS, Tucker. Robbins: Patologia Estrutural e Funcional. 6.ed. Rio de Janeiro:Guanabara Koogan,2000,p.441-458.

CRESPY,Vanessa; WILLIAMSON,Gary.A Review of the Health Effects of Green Tea Catechins in In Vivo Animal Models.Nestlé Research Center.Switzerland.2005.

CURTI, Fabiana; SaLGADO, Jocelem Mastrodi. Maçã: a fruta que cuida do nosso sangue. Nutrição Saúde e Performance: Anuário de Nutrição e Alimentos Funcionais; a. 6, n.26, trimestral, abr-mai-jun 2005, p. 6-11.

DEHOOG, Susan. Avaliação do Estado Nutricional. In: MAHAN, L. Kathleen; STUMP, Sylvia Escott. Krause: alimentos, nutrição e dietoterapia.9.ed.São Paulo:Roca,1998.

ELIAS, Maria Cristina; TEIXEIRA, Mônica. Leguminosas. Revista Nutrição Saúde e Performance: Anuário de Nutrição e Alimentos Funcionais.a.6.ed.26.trimestral. abr-maijun.São Paulo, 2005.p.47-51.

ELIAS, Maria Cristina;ITO, Mônica Teixeira;SLEIMAN, Júlia.Atualizações no Tratamento Dietoterápico nas Dislipidemias. Revista Nutrição Saúde e Performance:Anuário de Nutrição Clínica.a.3.n.13.out-nov.São Paulo,2001.

FARRET, Jacqueline Faria.Aplicações da Nutrição em Cardiologia.In: FARRET, Jacqueline Faria (coord.).Nutrição e doenças cardiovasculares:prevenção primária e secundária.São Paulo:Atheneu,2005.

FERNANDES,Ana Angélica Henrique et.al..Influência da dieta hipercalórica sobre parâmetros bioquímicos séricos, hepáticos e cardíacos em ratos-[s.l.],Nutrição em Pauta,bimestral,mar.-abr,São Paulo,2004. 
FERRARI, Carlos Kusano B..Bioquímica dos Alimentos Funcionais, Nutrição e Saúde.Revista Nutrição Profissional. a. 1, n.1, bimestral, maio-jun 2005, p 21-28.

GEBARA, Otávio. Colesterol em idosos:qual a importância. [s.l.], Cozinha Clássica Baixo Colesterol; a.1,n.5,mensal,set. 1999, p.4.

GÓES,Ângela. Alimentos funcionais: como separar o joio do trigo.Disponível em<http:// oglobo.globo.com/especiais/vivermelhor/mat/189692438.asp>Acesso em 20 fev 2006.

GÓES,Ângela.Alimentos Superpoderosos. Disponível em<http://oglobo.globo.com /especiais /vivermelhor/mat/189641004.asp> Acesso em 20 fev 2006.

GÓES,Ângela.Os alimentos ‘amigos do peito’.Disponível em<http://oglobo.globo.com/ especiais /vivermelhor/mat/189782115.asp > Acesso em 20 fev 2006.

GOLLUCKE, Andréa Pittelli Boiago; JUZWIAK, Cláudia Ridel. Chocolate Amargo: amigo do coração. Nutrição Saúde e Performance: Anuário de Nutrição e Alimentos Funcionais; a. 4, n.22, trimestral, jul-ago-set 2004, p.29-31.

GONÇALVES, Maria da Conceição Rodrigues.Berinjela: um alimento funcional. Nutrição Saúde e Performance: Anuário de Nutrição e Alimentos Funcionais; a. 4, n.22, trimestral, jul-ago-set 2004, p. 11-13.

GONZÁLEZ, M. et al. Effects of orange and apple pectin on cholesterol concentration in serum, liver and faeces. Jornal de Bioquímica.Espanha.jun.1998.

GUIMARÃES,P.R. et al. Eggplant (Solanum melongena) infusion has a modest and transitory effect on hypercholesterolemic subjects. São Paulo, 2000.

HONG,Jin Tae et al.. Protective effect of green tea extract on ischemia/ reperfusioninduced.South Korea.ago.2000

ISHIMOTO, Emilia Yasuko. Uva, Vinho e Qualidade de Vida. Nutrição Saúde e Performance: Anuário de Nutrição e Alimentos Funcionais; a. 4, n.22, trimestral, jul-agoset 2004, p. 53-55.

ISHIMOTO, Emilia Yasuko.Utilização de Alimentos Funcionais e Nutracêuticos na Prática Clínica: O Grande Desafio. Revista Nutrição Profissional. a. 1, n.1, bimestral, maio-jun 2005, p 29-37.

JORGE,Paulo Afonso Ribeiro et al. Efeito da Berinjela sobre os Lípides Plasmáticos, a Peroxidação Lipídica e a Reversão da Disfunção Endotelial na Hipercolesterolemia Experimental.Campinas.SP.2001. 
JUZWIAK, Cláudia Ridel. Prevenção de Dislipidemias Secundárias à Obesidade e Aterosclerose: os lipídeos na dieta da criança e adolescente. Revista Nutrição Saúde e Performance.a.3.n.13.out-nov.São Paulo,2001.

JUZWIAK, Cláudia Ridel; PASCHOAL, Valeria. Chá verde: prevenção e tratamento de doenças. Nutrição Saúde e Performance: Anuário de Nutrição e Alimentos Funcionais; a. 4, n.22, trimestral, jul-ago-set 2004.p.25-28.

JUZWIAK, Cláudia Ridel; PASCHOAL, Valéria. ENAF - Curso de Nutrição Funcional. Poços de Caldas - MG.maio.2003.

JUZWIAK, Cláudia Ridel; PASCHOAL, Valeria. O uso de suplementos no Tratamento das Dislipidemias e Prevenção da Doença Aterosclerótica.Nutrição Saúde e Performance: Anuário de Nutrição Clínica; trimestral,2004.p.18-19.

JUZWIAK, Cláudia Ridel; PASCHOAL, Valeria. Tomate e Goiaba. Nutrição Saúde e Performance: Anuário de Nutrição e Alimentos Funcionais; a. 4, n.22, trimestral, jul-agoset 2004, p. 50-52.

KRUMEL, Debra. Nutrição na Doença Cardiovascular. In: MAHAN, L. Kathleen; STUMP, Sylvia Escott. Krause: alimentos, nutrição e dietoterapia.10.ed.São Paulo:Roca,2002.

KUO KL; WENG MS; CHIANG CT; TSAI YJ; LIN-SHIAU SY; LIN JK. Comparative studies on the hypolipidemic and growth suppressive effects of oolong, black, pu-erh, and green tea leaves in rats. Jornal Agriculture Food. United States. 26 jan.2005.

LAIRON,D. et al.Dietary fiber intake and risk factors for cardiovascular disease in French adults. Jornal de Nutrição Clínica.2005.

LIBBY, Peter. A patogenia da aterosclerose.In:BRAUNWALD,Eugene et.al. Harrison: medicina interna.15.ed.v.1.São Paulo:Mc Graw Hill,2000.

LOPES, Cristina Garcia.Alimentos Funcionais - Parte II.Disponível em <http://www. cienciadoleite .com.br/alimfuncionais2.htm > Acesso em: 30 de abr de 2005.

MAESTÁ, Nailza;NAHAS, Eliana A. P.;BURINI, Roberto C..Alimentos Funcionais e Exercício Físico.Revista Nutrição em Pauta.bimestral.mar-abr.2006.p.16-20.

MARCÍLIO, Cláudia Stéfani. Nutrição para o Coração. Recomendações Nutricionais na Síndrome Metabólica. Revista Nutrição Profissional. a. 2, n.5, bimestral, jan-fev 2006, p 58 .

MARTENSEN, Ignez Campedelli. Cogumelos comestíveis: propriedades funcionais (Agaricus blazei e ?lentinus edodes). Revista Nutrição Saúde e Performance: Anuário de Alimentos Funcionais.a.6.ed.26.trimestral. abr-mai-jun.São Paulo, 2005.p.16-21. 
MELZIG,Matthias F. In vitro pharmacological activity of the tetrahydroisoquinoline salsolinol present in products from Theobroma cacao L. like cocoa and chocolate.

METZE, Konradin; MONTENEGRO, Mário Rubens. Artérias, Veias e Linfáticos. In: BRASILEIRO FILHO,Geraldo.Bogliolo Patologia.Rio de Janeiro:Guanabara Koogan,2000.

MOREIRA, Ana Vládia Bandeira. Especiarias: fontes importantes de compostos fenólicos antioxidantes. Nutrição Saúde e Performance: Anuário de Nutrição e Alimentos Funcionais; a. 4, n.22, trimestral, jul-ago-set 2004.p.32-34.

MOREIRA,Ana Vládia Bandeira;MANCINI FILHO,Jorge. Infleuncia dos compostos fenólicos de especiarias sobre a lipoperoxidação e o perfil lipídico de tecidos de ratos.Revista de Nutrição.v.17.n.4.trimestral.out-dez.2004.

MUKUDDEM, Peterson et al. Systematic rewiw of the effects of nuts on blood lipid profiles in humans. Jornal de Nutrição.Estados Unidos.2005.

NUNES,Maria Letícia Galluzzi. Inter-relações metabólicas: a lipogênese. UFRJ.Rio de Janeiro.2005.

OLIVEIRA, Alexandre Roberto Diogo de. Medicina Preventiva: saúde versus doenças.Rio de Janeiro:Biologia e Saúde,2002.

OLIVEIRA, Alexandre Roberto Diogo de; MONTEZ, Fabiano; GELINSKI, Paulo; SANTOS, Ricardo José dos. Medicina Preventiva: qualidade de vida.Rio de Janeiro:Biologia e Saúde,2002.

OLIVEIRA, Cláudia Pinto Marques Souza; LAUDANNA, Esther. Alimentação Saudável e Prevenção de Doenças. Revista Gastren.vol.15.n.2.2002.p 10-12

OLIVEIRA, Douglas Ferreira; ROSSETI, Lílian. Estudos Apontam Benefícios da Proteína Isolada de Soja.Revista Nutrição Profissional. a. 1, n.1, bimestral, maio-jun 2005, p 52-53.

OLIVEIRA, Silvana Pedroso.Alimentos funcionais: aspectos relacionados ao consumo. Disponível em http://www.revistafi.com.br/Main/revistas/ ed_20/ materias/ revisao.htm Acesso em 20 fev 2006.

PASCHOA, Marta Fonseca. Alimentação Escolar: Um Enfoque em Alimentos Funcionais.Revista Nutrição Profissional. a. 1, n.1, bimestral, maio-jun 2005, p 46-51. 
PASCHOAL, Valéria et al.Nutrição Clínica Funcional. Disponível em<http://www. vponline.com.br/edicao_conteudo_view.asp?id_tb_publicacao=1341> Acesso em 3 abr 2006.

PASCHOAL, Valéria. Alimentos para a saúde. Revista Sadia Light.dez.São Paulo,2001.

PASCHOAL, Valéria. Nutrientes Funcionais - Qualidade de Vida e Redução do Risco de Ocorrência de Doenças.Revista Nutrição Saúde e Performance.2003.p.8-9.

PASCHOAL, Valéria. Oleaginosas: potentes fontes de antioxidantes.Disponível em http://www.vponline.com.br/biblioteca_view.asp?id_tb_publicacao=385 Acesso em 18 abr 2006.

PIMENTEL,Carolina Vieira de Mello Barros; FRANCKI, Valeska Mangini Francki;GOLLÜCKE,Andréa Pittelli Boiago. Alimentos funcionais: introdução às principais substâncias bioativas em alimentos.São Paulo:Varela,2005.

PRAÇA, Juliana Marchiori; THOMAZ, Andréa; CARAMELLI,Bruno. O Suco de Berinjela (Solanum melongena) não Modifica os Níveis Séricos de Lípides Arquivos Brasileiros de Cardiologia .São Paulo.2004.

PRADO, F. Cintra;RAMOS, Jairo;VALLE, J. Ribeiro.Atualização Terapêutica.ed.20.São Paulo:Artes Médicas,2001.

QUINTAES,Diego Késia.Saiba mais sobre o Alho. Disponível em $<$ http://www.saudenarede.com.br/?p=av\&id=Saiba_mais_sobre_o_Alho> Acesso em 25 abr. 2006.

RISSANEN,T.H. et al.Serum lycopene concentrations and carotid atherosclerosis:the kuoplo ischaemic heart disease risk factor study.Jornal de Nutrição Clínica,2003.

ROMIJN,A. et al. Fish Oil increases bile acid synthesis in male Patients with hipertriglyceridemia. Jornal de Nutrição,2006.

SALGADO, Jocelem Mastrodi. Previna doenças: faça do alimento o seu medicamento. São Paulo:Madras, 2004.

SALGADO,Jocelem Mastrodi. SOCIEDADE Brasileira de Alimentos Funcionais.Disponível em<http://www.sbaf.org.br/sbaf/ >Acesso em 20 nov 2005.

SALGADO,Jocelem Mastrodi.Faça do alimento o seu medicamento.5.ed.São Paulo:MADRAS.2000.

SANTOS,Raul D. et al.III Diretrizes Brasileiras Sobre Dislipidemias e Diretriz de Prevenção da Aterosclerose. Sociedade Brasileira de Cardiologia.Arquivos Brasileiros de Cardiologia. v77.2001.Disponível em<http://www.cardiol.com> Acesso em 18 nov 2005. 
SILVA, Bianca Gerbassi de Carvalho. Alimentos Funcionais em cardiologia. In: FARRET, Jacqueline Faria (coord.).Nutrição e doenças cardiovasculares:prevenção primária e secundária.São Paulo:Atheneu,2005.

SOUSA,José Eduardo. News Letter: Como tratar a aterosclerose. Revista de Nutrição.n.9.out.2005.

SOUZA FILHO,Jairo Monson.O Vinho na medida certa.Disponível em $<$ http://www.nutricaoenoticias.com.br/magazine/?session=showNew\&id=5> Acesso em 05 maio 2006.

SOUZA, Rejane Dias das Neves. Soja e derivados: fatores que influenciam o teor de isoflavonas. Nutrição Saúde e Performance: Anuário de Nutrição e Alimentos Funcionais; a. 4, n.22, trimestral, jul-set 2004.p.44-49.

STUMP, Sylvia Escott; MAHAN, L. Kathleen. Krause: alimentos, nutrição e dietoterapia.9.ed.São Paulo:Roca,1998.

TAYLOR, Nadine.Is it true that Omega-3 fatty acids can protect the heart.University of Wisconsin-Madison School of Pharmacy.2005.Disponível em <http://www.ana-jana.org /reprints/janafishoilreprintforwebsite.pdf > Acesso em: 20 abr 2006.

TIRAPEGUI,Júlio.Nutrição: fundamentos e aspectos atuais.São Paulo:Atheneu,2002.

VIDAL, Luís Daniel Mariano;HANGUI, Maria Del Pilar T. Gómez;FATTAH, Tammuz. Lípides e doença aterosclerótica.Revista do Instituto Dante Pazzanense de Cardiologia.a.1.n.1.2001.

VIDAL,R. et al. Apple procyanidins decrease cholesterol esterification and lipoprotein secretion in Caco-2/TC7 enterocytes.França.fev.2005.

VIGGIANO, Celeste Elvira.A segunda era de Ouro da Nutrição: Alimentos Funcionais. Revista Nutrição Profissional. a. 1, n.1, bimestral, maio-jun 2005, p 12-20.

VIGGIANO, Celeste Elvira.Recomendações Nutricionais na Síndrome Metabólica. Revista Nutrição Profissional. a. 2, n.5, bimestral, jan-fev 2006, p 26-30.

WAN, Ying et al.. Effects of cocoa powder and dark chocolate on LDL oxidative susceptibilityand prostaglandin concentrations in humans. American Society for Clinical Nutrition .2001.

WILLIAM, H. Briggs et al. Administration of raw onion inhibits platelet-mediated thrombosis in dogs.jul.USA.2001. 
WOLLGAST,Jan;ANKLAM,Elke.Polyphenols in chocolate: is there a contribution to human health?. Institute for Health and Consumer Protection, Food Products and Consumer Goods Unit.Italy 3 fev. 2000.

ZACHÉ, Juliane;CASTELLÓN, Lena.Virada contra o colesterol. Revista Isto é. Disponible em <http://www.crestor.com.br/upload/imprensa/noticias_crestor/Crestor 2_Isto_E_02_06_04.doc>Acesso em 15 dez 2005.

ZBORAJ,Marian. Antioxidants: finding the right balance. Nutraceuticals World Magazine.mar.2006

\section{Informações bibliográficas:}

Conforme a NBR 6023:2002 da Associação Brasileira de Normas Técnicas (ABNT), este texto científico publicado em periódico eletrônico deve ser citado da seguinte forma:

BRAGA, A. A. D.; BARLETA, V. C. N. Alimento Funcional: Uma Nova Abordagem Terapêutica das Dislipidemias como Prevenção da Doença Aterosclerótica. Cadernos UniFOA, Volta Redonda, ano 2, nº. 3, mar. 2007. Disponível em: $<$ http://www.unifoa.edu.br/pesquisa/caderno/edicao/03/100.pdf> 